\title{
Anticuerpos conjugados a fármaco: la nueva generación de terapias biotecnológicas contra el cáncer
}

Guadalupe Melgarejo-Rubio, ${ }^{1}$ Sonia M. Pérez-Tapia, ${ }^{2}$ Emilio Medina-Rivero y Marco A. Velasco-Velázquez ${ }^{\text {* }}$ ${ }^{1}$ Universidad Nacional Autónoma de México, Facultad de Medicina, Departamento de Farmacología; 2 Instituto Politécnico Nacional, Escuela Nacional de Ciencias Biológicas, Unidad de Desarrollo e Investigación en Bioprocesos. Ciudad de México, México

\section{Resumen}

Los anticuerpos terapéuticos son proteínas recombinantes empleadas en el tratamiento del cáncer. Existe una nueva generación de anticuerpos monoclonales con actividad contra las células cancerosas, conocidos como anticuerpos conjugados a fármacos. Estas moléculas están integradas por tres elementos: un anticuerpo monoclonal, un fármaco citotóxico con alta potencia y un enlazador químico que los une. El anticuerpo reconoce antígenos tumorales, por lo que permite la entrega dirigida del agente citotóxico hacia las células cancerosas. Tras el reconocimiento de su antígeno, el anticuerpo conjugado a fármaco es endocitado por las células blanco, donde se induce la degradación lisosomal de la fracción proteica y se libera el fármaco citotóxico. En el presente artículo se revisan las características generales de los anticuerpos conjugados a fármacos y se describe la evidencia clínica de la eficacia y seguridad de los primeros cuatro aprobados por las agencias reguladoras de Estados Unidos y Europa.

PALABRAS CLAVE: Anticuerpos conjugados a fármacos. Gemtuzumab ozogamicina. Brentuximab vedotina. Trastuzumab emtansina. Inotuzumab ozogamicina.

\section{Antibody-drug conjugates: the new generation of biotechnological therapies against cancer}

\section{Abstract}

Therapeutic antibodies are recombinant proteins used in the treatment of cancer. There is a new generation of monoclonal antibodies with activity against cancer cells, known as antibody-drug conjugates. These molecules are made up of three elements: a monoclonal antibody, a highly potent cytotoxic drug, and a chemical linker that binds them together. The antibody recognizes tumor antigens, thereby allowing targeted delivery of the cytotoxic agent to cancer cells. After recognizing its antigen, the antibody-drug conjugate is endocytosed by the target cells, where the protein fraction is degradated into lysosomes, releasing the cytotoxic drug. This article reviews antibody-drug conjugates general characteristics and describes the clinical evidence of efficacy and safety of the first four approved by regulatory agencies in the United States and Europe.

KEY WORDS: Antibody-drug conjugates. Gemtuzumab ozogamicin. Brentuximab vedotin. Trastuzumab emtansine. Inotuzumab ozogamicin.

Correspondencia:

*Marco A. Velasco-Velázquez

E-mail: marcovelasco@unam.mx
Fecha de recepción: 03-10-2019

Fecha de aceptación: 23-01-2020

DOI: $10.24875 / G M M .20005572$
Gac Med Mex. 2020;156:229-236

Disponible en PubMed

www.gacetamedicademexico.com

0016-3813/@ 2020 Academia Nacional de Medicina de México, A.C. Publicado por Permanyer. Este es un artículo open access bajo la licencia CC BY-NC-ND (http://creativecommons.org/licenses/by-nc-nd/4.0/). 


\section{Generalidades de los anticuerpos terapéuticos}

Los anticuerpos son excelentes agentes que con alta especificidad inhiben moléculas concretas. El uso clínico de un anticuerpo se llevó a cabo por primera vez en 1986, después del desarrollo de la tecnología para producir anticuerpos monoclonales. Se trató de un anti-CD3 (muromonab) de origen murino cuyo uso, si bien era eficaz para reducir el rechazo a trasplantes, estuvo limitado por su rápida eliminación, su pobre capacidad para inducir funciones efectoras en el sistema inmune y por el desarrollo de anticuerpos contra el fármaco.

Para evitar esos problemas se han generado anticuerpos quiméricos, humanizados o totalmente humanos; $y$, mediante ingeniería de proteínas, los perfiles de solubilidad, agregación y estabilidad han sido mejorados para escalar su producción. En la actualidad existen anticuerpos recombinantes que pueden ser empleados en forma terapéutica. Esos productos, conocidos genéricamente como anticuerpos terapéuticos (AT), se utilizan en el tratamiento del cáncer y de otras enfermedades crónico-degenerativas o infecciosas. Por la incidencia de las enfermedades que pueden ser tratadas, la efectividad y posicionamiento en el mercado de los AT, estos constituyen el segmento de mayor crecimiento entre los productos biotecnológicos, con ventas que anualmente se incrementan en $5.3 \%$. $^{2}$ Tan solo en 2018, la Food and Drug Administration (FDA) aprobó 11 nuevos AT. ${ }^{3}$

\section{Los AT son inmunoglobulinas G}

Todos los AT en el mercado son inmunoglobulinas $G$ ( $\mathrm{IgG}$ que presentan estabilidad en la circulación, buena capacidad efectora y propiedades fisicoquímicas adecuadas para su formulación y administración. ${ }^{4}$ Las IgG son glucoproteínas compuestas por dos cadenas ligeras y dos cadenas pesadas (Figura 1A). La cadena ligera tiene dos dominios de inmunoglobulina: uno variable en el extremo $\mathrm{N}$-terminal $\left(\mathrm{V}_{\mathrm{L}}\right)$ y uno constante $\left(C_{L}\right)$. La cadena pesada tiene cuatro dominios de inmunoglobulina, uno variable en el extremo $\mathrm{N}$-terminal $\left(\mathrm{V}_{\mathrm{H}}\right)$ y tres constantes $\left(\mathrm{C}_{\mathrm{H}} 1, \mathrm{C}_{\mathrm{H}} 2\right.$ y $\left.\mathrm{C}_{\mathrm{H}} 3\right)$. La estructura del anticuerpo es estabilizada por enlaces disulfuro que unen cadenas ligeras con pesadas y pesadas entre sí, lo que le proporciona flexibilidad y una forma de " $Y$ " con dos fragmentos de unión de antígeno (Fab, fragment antigen-binding) idénticos. Cada Fab está

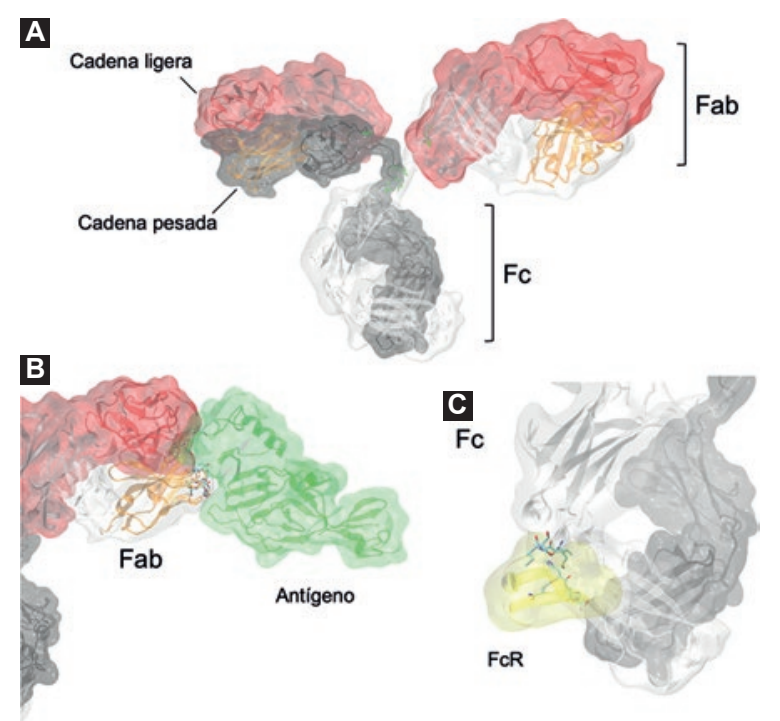

Figura 1. A) Representación de la estructura de una IgG: en rojo, las cadenas ligeras; en gris, las cadenas pesadas; en verde, los puentes disulfuro que las unen. La imagen se generó con información del Protein Data Bank (PDB, ID: 1IGT). B) Interacción de un fragmento de unión al antígeno (Fab) con un antígeno (verde). En esta región es donde recae la especificidad del anticuerpo. Imagen generada a partir de la información del PDB ID: 5KEL. C) Interacción de la región FC con un fragmento del FCR (amarillo). Otros sitios dentro de la región Fc pueden mediar la interacción con Cq1 o FcR. Imagen generada a partir de la información del PDB (ID: 1DN2).

formado por los dominios variables de una cadena pesada y una ligera (Figura 1B). La hipervariabilidad de las secuencias del Fab puede generar una diversidad casi infinita de anticuerpos con especificidad distinta. ${ }^{5}$

El fragmento de región cristalizable (región Fc) está formado únicamente por cadenas pesadas y desempeña un papel efector al unirse a receptores específicos $(\mathrm{FcR})$ expresados en células del sistema inmunitario, riñón o placenta (Figura 1C), o bien, al activar el complemento. En los seres humanos existen cuatro subclases de IgG con diferentes cadenas pesadas $(\gamma 1, \gamma 2$, $\gamma 3$, y $\gamma 4$ ), que si bien poseen alto grado de identidad $(90 \%)$, cada una tiene un perfil único ${ }^{6}$ respecto a:

- Capacidad de respuesta al antígeno.

- Activación de citotoxicidad dependiente de anticuerpo.

- Activación de citotoxicidad por complemento.

- Inducción de fagocitosis dependiente de anticuerpos.

- Tiempo de vida media.

- Transporte a través de la placenta.

La glucosilación de la región Fc induce cambios en la estructura cuaternaria que afectan sus funciones, por lo que es crucial conocer los patrones de glucosilación de las diferentes subclases y su relación con las propiedades funcionales del anticuerpo. ${ }^{7}$ La mayoría de 
$\mathbf{A}$

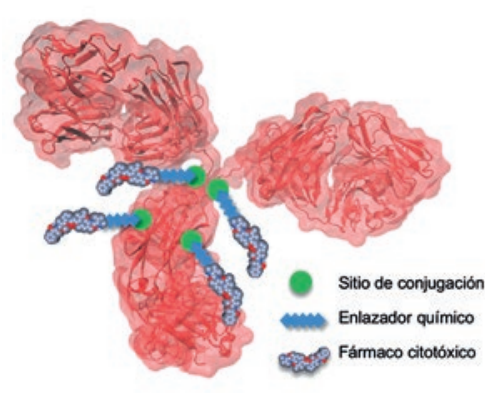

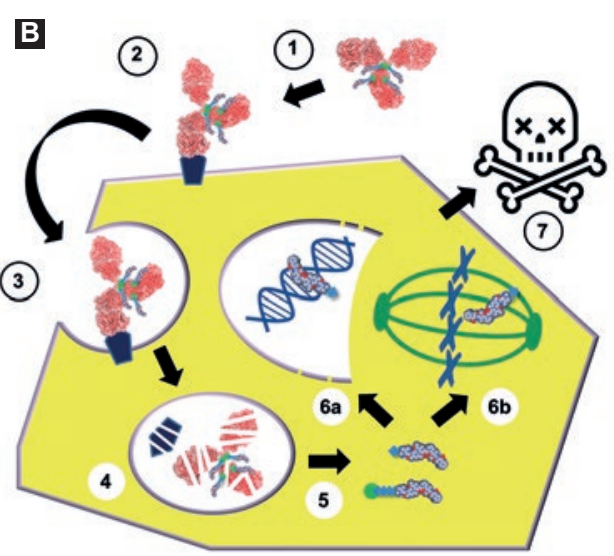

Figura 2. A) El ACF consta de tres elementos esenciales para su función: un anticuerpo (rojo), un fármaco citotóxico (gris) y un enlazador que une ambos (azul). Como sitio de conjugación (verde) puede usarse lisinas, cisteínas o aminoácidos modificados. B) La farmacocinética y farmacodinamia de los ACF son complejas e involucran la llegada del conjugado al tumor (1), la unión al antígeno tumoral (2), incorporación del complejo mediante endocitosis (3), la hidrólisis lisosomal de las proteínas (4), la liberación del fármaco citotóxico (5), la interacción del fármaco con su blanco intracelular, ya sea $\operatorname{ADN}(6 a)$, o tubulina (6b), y la inducción de la muerte de la célula (7).

los AT que se usan en las neoplasias son de isotipo IgG1, por su mayor capacidad para inducir citotoxicidad dependiente de anticuerpo o citotoxicidad por complemento en células que expresen el antígeno blanco. ${ }^{4}$

\section{Los anticuerpos conjugados, una nueva generación de fármacos antineoplásicos}

Los anticuerpos conjugados a fármacos (ACF) son anticuerpos monoclonales dirigidos contra antígenos tumorales, los cuales transportan fármacos citotóxicos a las células cancerosas, de ahí que los ACF se consideren profármacos. ${ }^{8}$ Los ACF tienen tres elementos centrales: el anticuerpo monoclonal acarreador, el fármaco citotóxico y el enlazador químico que permite la conjugación de ambos (Figura 2A). El anticuerpo permite el reconocimiento específico del antígeno en las células cancerosas. Después de la unión, el complejo ACFantígeno es incorporado en la célula tumoral mediante endocitosis y se induce su degradación lisosomal. Consecuentemente, el agente citotóxico es liberado dentro de la célula y desencadena la muerte de esta al interactuar con su blanco (Figura 2B). Este mecanismo aprovecha la especificidad del anticuerpo para depositar el citotóxico en el tejido tumoral, ${ }^{8}$ reduciendo la toxicidad sistémica y la posible resistencia.

\section{Características del antígeno y del anticuerpo}

El antígeno tumoral blanco dirige la biodistribución del ACF, por lo que debe sobreexpresarse en las células o en el microambiente tumoral y estar presente en niveles muy bajos o nulos en los tejidos sanos. ${ }^{9}$ Las proteínas de superficie celular son los blancos más empleados por su accesibilidad. Por ejemplo, la eficacia de los AT anti-HER2 está ligada a la elevada expresión del receptor, pues se han encontrado hasta dos millones de moléculas en la superficie de las células cancerosas. ${ }^{10}$ Sin embargo, en los linfomas de células B es suficiente que se expresen 30000 moléculas por célula del antígeno CD19 para que un ACF ejerza su efecto. ${ }^{11}$

Las células que no tienen en su superficie el antígeno de interés pueden estar expuestas al fármaco citotóxico si se encuentran cerca de las que son reconocidas por el ACF. Este mecanismo, conocido como "efecto del espectador inocente", es relevante cuando el anticuerpo identifica antígenos de la vasculatura o del estroma tumoral o cuando la expresión del antígeno no es homogénea en los tumores sólidos. ${ }^{12}$

Después de seleccionar el antígeno se requiere identificar el isotipo óptimo del anticuerpo, lo que influye en la eficacia, farmacocinética e índice terapéutico del ACF. La mayoría de los ACF que han alcanzado pruebas clínicas son de isotipo $\lg G 1 .^{9}$ Como se mencionó, las IgG1 inducen citotoxicidad dependiente de anticuerpo o mediada por complemento, por lo que se debe evaluar si se desea o no mantener estas funciones. En general, los anticuerpos empleados para generar ACF no deben desencadenar funciones efectoras. ${ }^{9}$ Sin embargo, cuando el anticuerpo que se conjugará tiene actividad terapéutica por sí mismo, puede ser relevante conservar 
dichas funciones. Los isotipos IgG2 e IgG4 son ineficientes para estimular respuestas inmunes secundarias. ${ }^{13}$ Las IgG2 permiten conjugar un número mayor de moléculas citotóxicas, dado que tienen cuatro puentes disulfuro en la región bisagra que pueden reducirse para la conjugación, mientras que lgG1 e IgG4 solo tienen dos. ${ }^{14}$ Aunque estudios comparativos han demostrado que los ACF conformados por anticuerpos IgG1 o IgG2 tienen perfiles de tolerabilidad y toxicidad similares,${ }^{15}$ las regiones bisagra de la IgG2 siguen siendo más difíciles de reducir. Por lo tanto, se ha optado por el diseño de IgG1 mutadas en la región Fc para atenuar su función efectora, ${ }^{15}$ pero conservando su capacidad de unirse al FcR para optimizar su vida media.

\section{Características de los enlazadores químicos}

El enlazador es una pieza fundamental de un ACF pues debe permitir la liberación del fármaco exclusivamente en el sitio de acción. Si el fármaco se libera en la circulación, se incrementa la toxicidad sistémica y disminuye la eficacia del ACF. Idealmente, el enlazador no debe interferir con la citotoxicidad del quimioterapéutico, dado que el subproducto citotóxico-enlazador es el que genera el efecto de espectador inocente. ${ }^{16}$ Además, el enlazador puede servir para contrarrestar la resistencia al fármaco citotóxico, ya que los enlazadores hidrofílicos generan metabolitos que no son sustratos para el eflujo o las bombas de expulsión activa de fármacos, como la glucoproteína $\mathrm{P}\left(\mathrm{P}\right.$-gp). ${ }^{17}$

En la actualidad se emplean dos tipos principales de enlazadores en el desarrollo de ACF: escindibles y no escindibles. ${ }^{17}$ Los enlazadores escindibles son estables durante el tiempo que están en circulación y liberan eficientemente el fármaco después de que el ACF es endocitado por la célula tumoral. Los enlazadores no escindibles permanecen unidos a un aminoácido del anticuerpo después de su degradación lisosomal. Este tipo de enlazadores es inadecuado para inducir el efecto de espectador inocente, pues el complejo aminoácido-enlazador-citotóxico no se difunde fuera de la célula tumoral. ${ }^{16}$

Ambos tipos de enlazadores aprovechan la reactividad de las lisinas o cisteínas en el anticuerpo para formar enlaces covalentes. La conjugación a lisinas es eficiente, pero genera múltiples especies de anticuerpos con diferencias en el número de moléculas del fármaco citotóxico y su localización. ${ }^{18}$ Adicionalmente, dado que muchas de las lisinas reactivas se encuentran en el dominio $\mathrm{C}_{\mathrm{H}} 2$, la conjugación incrementa la agregación del ACF.19 La conjugación a cisteínas requiere que estas se encuentren en su forma reducida, por lo que si se emplean las que forman puentes disulfuro intercatenarios, deben usarse enlazadores con dos grupos reactivos para permitir la regeneración del puente. Esta estrategia permite controlar mejor el número de moléculas del fármaco citotóxico que se conjuga, pero aun así pueden generarse más de 100 especies diferentes de ACF. ${ }^{18}$

La diversidad de especies afecta la estabilidad, farmacocinética y farmacodinamia del ACF. ${ }^{20}$ Por esto, se han desarrollado tecnologías que buscan incrementar la proporción de una especie particular, como la conjugación sitio-específica, la incorporación de nuevos residuos de cisteína o de aminoácidos no naturales o la conjugación enzimática. ${ }^{18}$

\section{Características de los fármacos citotóxicos}

El fármaco conjugado al anticuerpo es responsable de ejercer el efecto citotóxico en las células tumorales, por lo que debe cumplir con varias características (Yaghoubi et al. recientemente realizaron una revisión $\left.{ }^{21}\right)$ :

- Tener concentración inhibitoria media inferior al rango nanomolar, pues solo 1 a $2 \%$ del agente citotóxico alcanza su blanco intracelular.

- La unión covalente al enlazador químico no debe interferir en su actividad.

- Ser poco sensible al transporte mediado por P-gp para evitar la generación de resistencia.

- Tener propiedades fisicoquímicas que permitan su formulación para la administración intravenosa.

- Ser estable en el rango del pH que existe en el lisosoma.

Es difícil encontrar moléculas que cumplan con todas esas características, por lo que la mayoría de los ACF evaluados en humanos utilizan agentes de tres familias: caliqueamicinas, auristatinas o maitansinoides.

La caliqueamicina es un antitumoral aislado del actinomiceto Micromonospora echinospora. La caliqueamicina $\gamma 11$ es aproximadamente 1000 veces más potente que la doxorrubicina para inducir citotoxicidad. La N-acetil- $\gamma$-caliqueamicina, un análogo modificado, se emplea en los ACF. Las caliqueamicinas inducen muerte celular al unirse al surco menor del ADN, con preferencia por la secuencia TCCT/AGGA, ${ }^{22}$ formando especies birradicales que provocan la escisión de las hebras. ${ }^{23}$ Estos compuestos son hidrofóbicos, por lo que pocas moléculas pueden ser conjugadas al anticuerpo sin provocar agregación. ${ }^{9}$ 
Las auristatinas son análogos sintéticos de la dolastatina 10, un antimitótico aislado de la liebre marina Dolabella auricularia y posteriormente de cianobacterias. Las auristatinas generan un crecimiento continuo y excesivo de los microtúbulos al unirse a la subunidad $\beta$ de los dímeros de tubulina e impedir la hidrólisis del guanosín trifosfato; consecuentemente, se bloquea la separación de las cromátidas hermanas y la mitosis. ${ }^{24}$ Mientras que las concentraciones inhibitorias medias de otros fármacos que inhiben la polimerización de tubulina, como la vincristina o la vinblastina, se encuentran en el rango de $10^{-8}$ a $10^{-9} \mathrm{M}$, las auristatinas tienen concentraciones inhibitorias medias entre $10^{-10}$ y $10^{-12} \mathrm{M}^{-25}$. La monometil auristatina $E$ es una molécula sintética con propiedades fisicoquímicas optimizadas, por lo que ha sido utilizada en el desarrollo de múltiples ACF. ${ }^{26-29}$ Cuando la monometil auristatina $\mathrm{E}$ se conjuga al anticuerpo mediante enlazadores escindibles, el producto es lo suficientemente hidrofóbico para inducir el efecto de espectador inocente en las células vecinas. ${ }^{30}$

Los maitansinoides son sustancias citotóxicas derivadas de la maitansina, un antibiótico macrólido aislado del arbusto Maytenus ovatus. Se unen a la tubulina situada en los extremos de los microtúbulos, favoreciendo su despolimerización y llevando a la célula a apoptosis. ${ }^{31}$ Los maitansinoides han demostrado ser de 100 a 1000 veces más potentes in vitro que otros agentes citotóxicos; ${ }^{32}$ la emtansina y la mertansina se caracterizan por un sustituyente que contiene un grupo tiol, lo que facilita su conjugación a enlazadores. ${ }^{8}$ Estos compuestos tienen buena solubilidad y estabilidad en solución acuosa, pero pueden promover la agregación del ACF o limitar la unión al antígeno, especialmente a altos cocientes de conjugación. ${ }^{21}$

Otros citotóxicos en ACF empleados en ensayos clínicos incluyen doxorrubicina, ${ }^{33}$ pirrolobenzodiacepinas, ${ }^{34}$ indolinobenzodiacepinas, ${ }^{35}$ derivados de la camptotecina ${ }^{36}$ duocarmicinas ${ }^{37}$ y tubulisinas. ${ }^{38}$

\section{ACF disponibles}

Hasta mayo de 2019, cuatro ACF habían sido aprobados para su comercialización por la FDA y la European Medicines Agency (EMA) cada uno se describe a continuación.

\section{Gemtuzumab ozogamicina}

Gemtuzumab ozogamicina es una IgG4 humanizada anti-CD33 conjugada a $\mathrm{N}$-acetil- $\gamma$-caliqueamicina mediante un enlazador bifuncional. ${ }^{39,40}$ CD33 es una molécula de adhesión celular perteneciente a la superfamilia de lectinas que se unen al ácido siálico, ${ }^{41}$ la cual se expresa en las células mieloides y en aproximadamente 85 a $90 \%$ de los pacientes con leucemia mieloide aguda.

En el año 2000, gemtuzumab ozogamicina fue el primer ACF en ser aprobado por la FDA para el tratamiento de pacientes con leucemia mieloide aguda CD33+ en recaída, con 60 años o más y que no eran candidatos para otras quimioterapias. Sin embargo, en el periodo de poscomercialización se demostró su falta de eficacia y su asociación con efectos secundarios graves y muerte prematura: la adición de gemtuzumab ozogamicina a la quimioterapia estándar de inducción (daunorrubicina + citarabina) no mejoró la tasa de respuestas completas ni la supervivencia libre de recaída, ${ }^{42}$ incrementó la mortalidad de 1.4 a $5.5 \%{ }^{42}$ y la incidencia de enfermedad venooclusiva hepática fue de aproximadamente $10 \%{ }^{43}$ Por esos motivos, el fármaco fue retirado del mercado en 2010.

Se han planteado diversas hipótesis para explicar el fracaso del producto: la pobre estabilidad del enlazador químico, la heterogeneidad en la cantidad de moléculas del fármaco unidas al anticuerpo y la susceptibilidad del agente citotóxico para ser acarreado por transportadores. ${ }^{9,17} \mathrm{Al}$ ser poco inmunogénico, no se identificó la relación de los anticuerpos anti-ACF con la eficacia y seguridad de gemtuzumab ozogamicina ${ }^{44}$

Ensayos posteriores demostraron que dosis más bajas y frecuentes incrementaban la eficacia y seguridad del gemtuzumab ozogamicina. En adultos con leucemia mieloide aguda de reciente manifestación, la adición de ACF a la quimioterapia incrementó la supervivencia libre de eventos a los dos años, así como la supervivencia global. ${ }^{40}$ Como monoterapia, favorece la obtención de respuestas completas en los niños con leucemia mieloide aguda refractaria o reincidente. ${ }^{39}$ Estos resultados contribuyeron a que gemtuzumab fuera aprobado en 2017 por la FDA para el tratamiento de pacientes que presentan leucemia mieloide aguda CD33+ durante la edad adulta y de niños con recaída o que no responden al tratamiento primario. ${ }^{45}$ Meses después fue aprobado por la EMA. ${ }^{46}$ Aún se reportan efectos secundarios, aunque menos graves. ${ }^{40,45}$

\section{Brentuximab vedotina}

Brentuximab vedotina es una IgG1 quimérica anti-CD30 conjugada a monometil auristatina $E$ 
mediante un enlazador escindible de valina-citrulina sensible a la catepsina B. ${ }^{47-49}$ CD30 pertenece a la familia de receptores del factor de necrosis tumoral y normalmente se expresa en tejidos linfoides y se sobreexpresa en células neoplásicas de pacientes con linfoma de Hodgkin, linfoma anaplásico de células grandes o linfoma cutáneo de células T.50

En los pacientes que recaen en linfoma de Hodgkin, sin respuesta al trasplante autólogo de células troncales, la monoterapia con brentuximab vedotina mejora las tasas de respuesta completa, así como la supervivencia libre de progresión a cinco años y la supervivencia global. ${ }^{47-49} \mathrm{En}$ pacientes con linfoma de Hodgkin sin tratamiento previo, la combinación de brentuximab vedotina con doxorrubicina, vinblastina y dacarbazina es igualmente eficaz y menos tóxica que la adición de un cuarto agente quimioterapéutico al esquema terapéutico. ${ }^{51,52}$ Brentuximab vedotina ha demostrado beneficios cuando se usa como monoterapia en pacientes con linfoma anaplásico de células grandes ${ }^{53}$ o linfoma cutáneo de células $T,{ }^{54}$ ya que aumenta significativamente la tasa de respuesta global y la supervivencia libre de progresión.

Brentuximab vedotina induce reacciones adversas, especialmente en pacientes con alteraciones en la función hepática 0 renal ${ }^{55} \mathrm{o}$ que consumen fármacos que inhiben el CYP3A4, pues la monometil auristatina E se metaboliza por esa isoforma del citocromo P450. ${ }^{56}$

\section{Trastuzumab emtansina}

Trastuzumab emtansina es una IgG1 humanizada anti-HER2 conjugada a emtansina mediante un enlazador tioéter no escindible unido a residuos de lisina. ${ }^{57,58}$ HER2 se sobreexpresa en un subgrupo de tumores mamarios, por lo que trastuzumab se desarrolló como AT. ${ }^{59}$ Se conocen bien la farmacocinética, farmacodinamia y reacciones adversas del trastazumab. ${ }^{59}$ Trastuzumab emtansina conserva los mecanismos de acción propios del anticuerpo sin conjugar, además de inducir citotoxicidad por emtansina. ${ }^{60}$

En los pacientes con cáncer de mama HER2+ localmente avanzado, irresecable o metastásico previamente tratados, trastuzumab emtansina es más efectivo que la combinación de capecitabina + lapatinib ${ }^{57}$ o un tratamiento de tercera línea, ${ }^{58}$ pues aumenta la supervivencia libre de progresión y la mediana de la supervivencia global. Además, las reacciones adversas fueron mejor toleradas en los grupos tratado con el ACF. ${ }^{57,58}$ En contraste, en los pacientes con cáncer de mama avanzado HER2+ sin tratamiento previo, trastuzumab emtansina no es más eficaz que trastuzumab combinado con algún taxano, ${ }^{61}$ por lo que la aprobación de su uso se limita a pacientes previamente tratados.

Existen reportes de desarrollo de resistencia a este ACF. Esto puede deberse a lo siguiente: ${ }^{62}$

- Mecanismos de resistencia a trastuzumab.

- Incorporación ineficiente del ACF.

- Degradación lisosomal reducida.

- Acción de endosomas de reciclaje que permiten el regreso del complejo HER2-ACF a la membrana.

Aunque $5 \%$ de los pacientes que reciben trastuzumab emtansina desarrollan anticuerpos contra este agente, ${ }^{44}$ ello no impacta en su eficacia.

Se ha registrado que trastuzumab emtansina induce múltiples respuestas adversas; la trombocitopenia es la más grave y la que motiva que se limite la dosis. ${ }^{63}$ Al igual que trastuzumab, puede inducir disfunción ventricular izquierda o falla hepática. ${ }^{63}$ Estos efectos se incrementan por el consumo de fármacos que interfieren con el metabolismo de emtansina. ${ }^{62}$

\section{Inotuzumab ozogamicina}

Inotuzumab ozogamicina es una IgG4 humanizada anti-CD22 conjugada a $\mathrm{N}$-acetil- $\gamma$-caliqueamicina mediante el mismo enlazador bifuncional empleado en gemtuzumab ozogamicina. ${ }^{64-66}$ CD22 es una glucoproteína transmembranaria de la superfamilia de lectinas que se unen al ácido siálico ${ }^{41}$ la cual se sobreexpresa en más de $90 \%$ de los pacientes con leucemia linfoblástica aguda. ${ }^{67}$ Inotuzumab ozogamicina se emplea como monoterapia para el tratamiento de adultos con leucemia linfoblástica aguda CD22+ recidivante o refractaria. En esos pacientes, el uso de ACF incrementa significativamente la tasa de pacientes con remisión completa, la supervivencia global a dos años y la calidad de vida. ${ }^{64-66}$

El enlazador en inotuzumab ozogamicina es estable en la circulación sistémica, ${ }^{68}$ aun cuando se le atribuye la toxicidad de otros ACF. Inotuzumab ozogamicina induce efectos adversos que se pueden mitigar con medidas preventivas y monitoreo constante de los signos y síntomas. ${ }^{69}$ Aunque $3 \%$ de los pacientes que reciben inotuzumab ozogamicina genera anticuerpos contra el producto, aún no se ha determinado el impacto de esta reacción en la eficacia y seguridad del agente. ${ }^{44}$

\section{Conclusiones y perspectivas}

Aunque los ACF representan un gran avance en oncología, tienen diversas limitaciones, por ejemplo, 
aun cuando un ACF sea el indicado para un tipo de tumor, su utilidad se circunscribe a subgrupos específicos de pacientes. Por lo anterior, se requiere analizar el efecto de los ACF en diferentes poblaciones y tipos de tumores que expresen el antígeno blanco. Un ejemplo de ese tipo de análisis es el relativo a la eficacia de trastuzumab emtansina en cáncer de pulmón. ${ }^{70}$ Paralelamente, deben estudiarse los mecanismos por los que se desarrolla resistencia a los ACF, con la finalidad de proponer estrategias que permitan revertirla. El eflujo de fármacos citotóxicos es un factor importante en la resistencia, por lo que se ha propuesto combinar el ACF con inhibidores de los transportadores que permiten ese mecanismo ${ }^{71} 0$ generar nuevos agentes que no sean transportados. ${ }^{21}$ También son necesarias más investigaciones sobre la frecuencia con la que se desarrollan anticuerpos antiACF y su papel en el desarrollo de resistencia. ${ }^{44}$

Aún debe mejorarse la seguridad de los ACF. Generalmente, los efectos adversos son causados por la inducción de la muerte de células sanas. Esa citotoxicidad podría deberse a la expresión del antígeno blanco en las células, a que estas capten el conjugado de forma independiente al antígeno o a que el fármaco citotóxico sea liberado a la circulación a partir de células blanco que procesaron el ACF.

Es de esperar que en el futuro cercano se desarrollen nuevos ACF que sean más efectivos y seguros, ya que existe suficiente información respecto a cuál debe ser la naturaleza del antígeno seleccionado, qué propiedades debe tener el anticuerpo, cuáles son las propiedades farmacológicas y fisicoquímicas indispensables del agente citotóxico y cómo debe funcionar el enlazador. Los ACF incrementarán las oportunidades de supervivencia y la calidad de vida de los pacientes con cáncer.

\section{Agradecimientos}

Agradecemos al doctor Luis Córdoba Bahena, la revisión crítica del manuscrito y la realización de las figuras.

\section{Conflicto de intereses}

Ninguno.

\section{Financiamiento}

Para la realización de esta investigación se recibió financiamiento del Consejo Nacional de Ciencia y Tecnología (proyecto A1-S-18285), así como apoyo del
Laboratorio Nacional para Servicios Especializados de Investigación, Desarrollo e Innovación para Farmoquímicos y Biotecnológicos (LANSEIDI-FarBiotecCONACYT).

\section{Responsabilidades éticas}

Los autores declaran que para esta investigación no se realizaron experimentos en seres humanos ni en animales.

Confidencialidad de los datos. Los autores declaran que en este artículo no aparecen datos de pacientes.

\section{Bibliografía}

1. Liu JKH. The history of monoclonal antibody development - Progress, remaining challenges, and future innovations. Ann Med Surg (Lond). 2014;3:113-116.

2. Elvin JG, Couston RG, van der-Walle CF. Therapeutic antibodies: Market considerations, disease targets and bioprocessing. Int J Pharm. 2013;440:83-98.

3. Mullard A. 2018 FDA drug approvals. Nat Rev Drug Discov. 2019;18:85-89.

4. Goulet DR, Atkins WM. Considerations for the design of antibody-based therapeutics. J Pharm Sci. 2020;109:74-103.

5. Backhaus O. Generation of antibody diversity. En: Böldicke T, editor. Antibody engineering. México: InTech; 2018. DOI: 10.5772/65238

6. Vidarsson G, Dekkers G, Rispens T. IgG subclasses and allotypes: from structure to effector functions. Front Immunol. 2014;5:520.

7. Cobb BA. The history of IgG glycosylation and where we are now. Glycobiology. 2020;30(4):202-213

8. Dan N, Setua S, Kashyap VK, Khan S, Jaggi M, Yallapu MM, et al. Antibody-drug conjugates for cancer therapy: chemistry to clinical implications. Pharmaceuticals (Basel). 2018;11(2). pii: E32

9. Beck A, Goetsch L, Dumontet C, Corvaïa N. Strategies and challenges for the next generation of antibody-drug conjugates. Nat Rev Drug Discov. 2017:16:315-337.

10. Horton J. Her2 and trastuzumab in breast cancer. Cancer Control. 2001;8:103-110.

11. Blanc V, Bousseau A, Caron A, Carrez C, Lutz RJ, Lambert JM. SAR3419: An anti-CD19-Maytansinoid Immunoconjugate for the treatment of B-cell malignancies. Clin Cancer Res. 2011;17:6448-6458.

12. Damelin M, Zhong W, Myers J, Sapra P. Evolving strategies for target selection for antibody-drug conjugates. Pharm Res. 2015;32:3494-3507.

13. Kretschmer A, Schwanbeck R, Valerius $T$, Rösner $T$. Antibody isotypes for tumor immunotherapy. Transfus Med Hemother. 2017;44:320-326.

14. Wiggins B, Liu-Shin L, Yamaguchi H, Ratnaswamy G. Characterization of cysteine-linked conjugation profiles of immunoglobulin $\mathrm{G} 1$ and immunoglobulin G2 antibody-drug conjugates. J Pharm Sci. 2015;104:1362-1372.

15. McDonagh CF, Kim KM, Turcott E, Brown LL, Westendorf L, Feist T, et al. Engineered anti-CD70 antibody-drug conjugate with increased therapeutic index. Mol Cancer Ther. 2008;7:2913-2923.

16. Kovtun YV, Goldmacher VS. Cell killing by antibody-drug conjugates. Cancer Lett. 2007;255:232-240.

17. Tsuchikama K, An Z. Antibody-drug conjugates: recent advances in conjugation and linker chemistries. Protein Cell. 2018;9:33-46.

18. Panowski $S$, Bhakta $S$, Raab $H$, Polakis $P$, Junutula JR. Site-specific antibody drug conjugates for cancer therapy. MAbs. 2014;6:34-45.

19. Wakankar AA, Feeney MB, Rivera J, Chen Y, Kim M, Sharma VK, et al. Physicochemical stability of the antibody-drug conjugate Trastuzumab-DM1: changes due to modification and conjugation processes. Bioconjug Chem. 2010;21:1588-1595.

20. Boylan NJ, Zhou W, Proos RJ, Tolbert TJ, Wolfe JL, Laurence JS Conjugation site heterogeneity causes variable electrostatic properties in Fc conjugates. Bioconjug Chem. 2013;24:1008-1016.

21. Yaghoubi S, Karimi MH, Lotfinia M, Gharibi T, Mahi-Birjand M, Kavi E, et al. Potential drugs used in the antibody-drug conjugate (ADC) architecture for cancer therapy. J Cell Physiol. 2020;235:31-64.

22. Ellestad GA. Structural and conformational features relevant to the anti-tumor activity of calicheamicin $\gamma 11$. Chirality. 2011;23:660-671.

23. Zein N, Sinha AM, McGahren WJ, Ellestad GA. Calicheamicin gamma 1l: an antitumor antibiotic that cleaves double-stranded DNA site specifically. Science. 1988;240:1198-1201. 
24. Chen H, Lin Z, Arnst K, Miller D, Li W. Tubulin inhibitor-based antibody-drug conjugates for cancer therapy. Molecules. 2017;22:1281.

25. Bai R, Pettit GR, Hamel E. Dolastatin 10, a powerful cytostatic peptide derived from a marine animal. Inhibition of tubulin polymerization mediated through the vinca alkaloid binding domain. Biochem Pharmacol. 1990;39:1941-1949

26. Li Z, Wang M, Yu D, Luo W, Fang J, Huang C, et al. Monomethyl auris tatin E-conjugated anti-EGFR antibody inhibits the growth of human EGFR-positive non-small cell lung cancer. Cancer Chemother Pharmacol. 2019;84:61-72

27. Lütje S, Gerrits D, Molkenboer-Kuenen JD, Herrmann K, Fracasso G, Colombatti $\mathrm{M}$, et al. Characterization of site-specifically conjugated monomethyl auristatin E- and duocarmycin-based anti-PSMA antibody-drug conjugates for treatment of PSMA-expressing tumors. J Nucl Med. 2018:59:494-501.

28. Bourillon L, Bourgier C, Gaborit N, Garambois V, Llès E, Zampieri A et al. An auristatin-based antibody-drug conjugate targeting HER3 enhances the radiation response in pancreatic cancer. Int $\mathrm{J}$ Cancer. 2019:145:1838-1851.

29. Tilly H, Morschhauser F, Bartlett NL, Mehta A, Salles G, Haioun C, et al. Polatuzumab vedotin in combination with immunochemotherapy in patients with previously untreated diffuse large B-cell lymphoma: an open-label, non-randomised, phase 1b-2 study. Lancet Oncol. 2019;20:998-1010.

30. Okeley NM, Miyamoto JB, Zhang X, Sanderson RJ, Benjamin DR, Sievers EL, et al. Intracellular activation of SGN-35, a potent anti-CD30 antibody-drug conjugate. Clin Cancer Res. 2010;16:888-897.

31. Lopus M, Oroudjev E, Wilson L, Wilhelm S, Widdison W, Chari R, et al. Maytansine and cellular metabolites of antibody-maytansinoid conjugates strongly suppress microtubule dynamics by binding to microtubules. Mol Cancer Ther. 2010;9:2689-2699.

32. Widdison WC, Wilhelm SD, Cavanagh EE, Whiteman KR, Leece BA, Kovtun $Y$, et al. Semisynthetic maytansine analogues for the targeted treatment of cancer. J Med Chem. 2006;49:4392-4408.

33. Govindan SV, Cardillo TM, Sharkey RM, Tat F, Gold DV, Goldenberg DM. Milatuzumab-SN-38 conjugates for the treatment of CD74+ cancers. Mol Cancer Ther. 2013;12:968-978.

34. Mantaj J, Jackson PJM, Rahman KM, Thurston DE. From anthramycin to pyrrolobenzodiazepine (PBD)-containing antibody-drug conjugates (ADCs). Angew Chem Int Ed Engl. 2017:56:462-488.

35. Miller ML, Fishkin NE, Li W, Whiteman KR, Kovtun Y, Reid EE, et al. A New class of antibody-drug conjugates with potent DNA alkylating activity. Mol Cancer Ther. 2016;15:1870-1878.

36. Nabada T, Masuda T, Naito H, Yoshida M, Ashida S, Morita K, et al. Novel antibody drug conjugates containing exatecan derivative-based cytotoxic payloads. Bioorg Med Chem Lett. 2016;26:1542-1545.

37. Elgersma RC, Coumans RGE, Huijbregts T, Menge WMPB, Joosten JAF Spijker HJ, et al. Design, synthesis, and evaluation of linker-duocarmycin payloads: toward selection of HER2-targeting antibody-drug conjugate SYD985. Mol Pharm. 2015;12:1813-1835.

38. Li JY, Perry SR, Muñiz-Medina V, Wang X, Wetzel LK, Rebelatto MC et al. A biparatopic HER2-targeting antibody-drug conjugate induces tumor regression in primary models refractory to or ineligible for HER2-targeted therapy. Cancer Cell. 2016;29:117-129.

39. Zwaan CM, Reinhardt D, Zimmerman M, Hasle H, Stary J, Stark B, et al. Salvage treatment for children with refractory first or second relapse of acute myeloid leukaemia with gemtuzumab ozogamicin: Results of a phase II study. Br J Haematol. 2010;148:768-776.

40. Catarine S, Pautas C, Terré C, Raffoux E, Bordessoule D, Bastie JN, et al. Effect of gemtuzumab ozogamicin on survival of adult patients with de-novo acute myeloid leukaemia (ALFA-0701): A randomised, open-label, phase 3 study. Lancet. 2012;379:1508-1516.

41. Läubli H, Varki A. Sialic acid-binding immunoglobulin-like lectins (Siglecs) detect self-associated molecular patterns to regulate immune responses. Cell Mol Life Sci. 2020;77:593-605

42. Petersdorf SH, Kopecky KJ, Slovak M, Willman C, Nevill T, Brandwein J, et al. A phase 3 study of gemtuzumab ozogamicin during induction and postconsolidation therapy in younger patients with acute myeloid leukemia. Blood. 2013;121:4854-4860

43. Tack DK, Letendre L, Kamath PS, Tefferi A. Development of hepatic veno-occlusive disease after mylotarg infusion for relapsed acute myeloid leukemia. Bone Marrow Transplant. 2001;28:895-897.

44. Carrasco-Triguero M, Dere RC, Milojic-Blair M, Saad OM, Nazzal D, Hong K, et al. Immunogenicity of antibody-drug conjugates: observations across eight molecules in eleven clinical trials. Bioanalysis. 2019;11:1555-1568.

45. Appelbaum FR, Bernstein ID. Gemtuzumab ozogamicin for acute myeloid leukemia. Blood. 2017:130:2373-2376.

46. Ali S, Dunmore H-M, Karres D, Hay JL, Salmonsson T, Gisselbrecht C et al. The EMA review of mylotarg (gemtuzumab ozogamicin) for the treatment of acute myeloid leukemia. Oncologist. 2019;24:e171-e179.

47. Younes A, Gopal AK, Smith SE, Ansell SM, Rosenblatt JD, Savage KJ, et al. Results of a pivotal phase II study of brentuximab vedotin for patients with relapsed or refractory Hodgkin's lymphoma. J Clin Oncol. 2012;30:2183-2189.
48. Chen R, Gopal AK, Smith SE, Ansell SM, Rosenblatt JD, Savage KJ, et al. Five-year survival and durability results of brentuximab vedotin in patients with relapsed or refractory Hodgkin lymphoma. Blood. 2018;128:1562-1566.

49. Moskowitz $\mathrm{CH}$, Nademanee A, Masszi T, Agura E, Holowiecki J, Abidi $\mathrm{MH}$, et al. Brentuximab vedotin as consolidation therapy after autologous stem-cell transplantation in patients with Hodgkin's lymphoma at risk of relapse or progression (AETHERA): a randomised, double-blind, placebo-controlled, phase 3 trial. Lancet. 2015:385:1853-1862.

50. Chiarle R, Podda A, Prolla G, Gong J, Thorbecke GJ, Inghirami G. CD30 in normal and neoplastic cells. Clin Immunol. 1999;90:157-164.

51. Younes A, Connors JM, Park SI, Fanale M, O'Meara MM, Hunder NN et al. Brentuximab vedotin combined with ABVD or AVD for patients with newly diagnosed Hodgkin's lymphoma: a phase 1, open-label, dose-escalation study. Lancet Oncol. 2013;14:1348-1356.

52. Connors JM, Jurczak W, Straus DJ, Ansell SM, Kim WS, Gallamini A, et al. Brentuximab vedotin with chemotherapy for stage III or IV Hodgkin's lymphoma. N Engl J Med. 2018;378:331-344.

53. Pro B, Advani R, Brice $P$, Bartlett NL, Rosenblatt JD, Illidge $T$, et al. Five-year results of brentuximab vedotin in patients with relapsed or refractory systemic anaplastic large cell lymphoma. Blood. 2017;130:2709-2717.

54. Prince HM, Kim YH, Horwitz SM, Dummer R, Scarisbrick J, Quaglino P, et al. Brentuximab vedotin or physician's choice in CD30-positive cutaneous T-cell lymphoma (ALCANZA): an international, open-label, randomised, phase 3, multicentre trial. Lancet. 2017;390:555-566.

55. Oak E, Bartlett NL. A safety evaluation of brentuximab vedotin for the treatment of Hodgkin lymphoma. Expert Opin Drug Saf. 2016;15:875-882.

56. Han TH, Gopal AK, Ramchandren R, Goy A, Chen R, Matous JV, et al. CYP3A-mediated drug-drug interaction potential and excretion of brentuximab vedotin, an antibody-drug conjugate, in patients with CD30-positive hematologic malignancies. J Clin Pharmacol. 2013;53:866-877.

57. Verma S, Miles D, Gianni L, Krop IE, Welslau M, Baselga J, et al. Trastuzumab emtansine for HER2-positive advanced breast cancer. N Engl J Med. 2012;367:1783-1791.

58. Krop IE, Kim SB, González-Martín A, LoRusso PM, Ferrero J-M, Smitt M, et al. Trastuzumab emtansine versus treatment of physician's choice for pretreated HER2-positive advanced breast cancer (TH3RESA): a randomised, open-label, phase 3 trial. Lancet Oncol. 2014;15:689-699.

59. Lambertini M, Pondé NF, Solinas C, de Azambuja E. Adjuvant trastuzumab: a 10-year overview of its benefit. Expert Rev Anticancer Ther. 2017:17:61-74.

60. Junttila TT, Li G, Parsons K, Phillips GL, Sliwkowski MX. Trastuzumab-DM1 (T-DM1) retains all the mechanisms of action of trastuzumab and efficiently inhibits growth of lapatinib insensitive breast cancer. Breast Cancer Res Treat. 2011;128:347-356.

61. Perez EA, Barrios C, Eiermann W, Toi M, Im YH, Conte P, et al. Trastuzumab emtansine with or without pertuzumab versus trastuzumab plus taxane for human epidermal growth factor receptor 2-positive, advanced breast cancer: primary results from the phase III MARIANNE study. J Clin Oncol. 2017;35:141-148.

62. Barok M, Joensuu H, Isola J. Trastuzumab emtansine: Mechanisms of action and drug resistance. Breast Cancer Res. 2014;16:209.

63. Kowalczyk L, Bartsch R, Singer CF, Farr A. Adverse events of trastuzumab emtansine (T-DM1) in the treatment of HER2-positive breast cancer patients. Breast Care (Basel). 2017;12:401-408

64. Kantarjian HM, DeAngelo DJ, Stelljes M, Martinelli G, Liedtke M, Stock $W$, et al. Inotuzumab ozogamicin versus standard therapy for acute lymphoblastic leukemia. N Engl J Med. 2016:375:740-753.

65. Kantarjian HM, DeAngelo DJ, Stelljes M, Liedtke M, Stock W, Gökbuget N, et al. Inotuzumab ozogamicin versus standard of care in relapsed or refractory acute lymphoblastic leukemia: Final report and long-term survival follow-up from the randomized, phase 3 INO-VATE study. Cancer. 2019;125:2474-2487.

66. Su Y, van Oostrum I, Vandendries E, Welch V, Loberiza FR. Hospitalization for patients in the United States (US) and European Union (EU) treated with inotuzumab ozogamicin (InO) vs standard of care (SOC) for relapsed/refractory acute lymphoblastic leukemia (R/R ALL) in a global phase 3 trial. J Clin Oncol. 2017;35:e18500-e18500.

67. Shah NN, Stevenson MS, Yuan CM, Richards K, Delbrook C, Kreitman RJ, et al. Characterization of CD22 expression in acute lymphoblastic leukemia. Pediatr Blood Cancer. 2015:62:964-969.

68. Boghaert ER, Khandke KM, Sridharan L, Dougher M, DiJoseph JF, Kunz A, et al. Determination of pharmacokinetic values of calicheamicin-antibody conjugates in mice by plasmon resonance analysis of small ( 5 microl) blood samples. Cancer Chemother Pharmacol. 2008:61:1027-1035.

69. Kebriaei P, Cutler C, de Lima M, Giralt S, Lee SJ, Marks D, et al. Management of important adverse events associated with inotuzumab ozogamicin: expert panel review. Bone Marrow Transplant. 2018:53:449-456.

70. Ohashi K, Hotta K, Hirata T, Aoe K, Kozuki T, Ninomiya K, et al. Trastuzumab emtansine in HER2+ recurrent metastatic non-small-cell lung cancer: study protocol. Clin Lung Cancer. 2017;18:92-95.

71. Chang $\mathrm{CH}$, Wang $\mathrm{Y}$, Zalath M, Liu D, Cardillo TM, Goldenberg DM. Combining ABCG2 inhibitors with IMMU-132, an anti-Trop-2 antibody conjugate of SN-38, overcomes resistance to SN-38 in breast and gastric cancers. Mol Cancer Ther. 2016;15:1910-1919. 